\title{
Stability of a condensing liquid film in a binary vapor mixture system
}

\author{
Kentaro Kanatani* \\ Faculty of Engineering, Yokohama National University, \\ 79-5 Tokiwadai, Hodogaya, Yokohama 240-8501, Japan
}

\begin{abstract}
We study stability of a condensing liquid film of a binary vapor mixture. When a binary vapor mixture of some kind is cooled on a substrate, a condensing liquid film emerges to take an inhomogeneous form such as a droplet one due to the solutal Marangoni effect. In order to analyze this phenomenon, we apply the long-wave approximation to the condensing liquid film and derive a nonlinear partial differential equation describing the spatio-temporal evolution of the film thickness. An interfacial boundary condition taking account of an effect of mass gain of the liquid film is adopted. Based on this model, we perform a linear stability analysis around a flat-film solution. We obtain an evolution equation of the amplitude of a disturbance, from which the cutoff and fastest growth wavenumbers are deduced. The maximum value of the cutoff wavenumber relative to the film thickness and its film thickness are estimated for water-ethanol mixture at atmospheric pressure. We numerically verify the long-wave nature of the instability of the condensate liquid film in this system. A significant difference in their values is found for low-ethanol fractions of the ambient vapor whether or not the temperature dependence of the mass transfer coefficient is considered. The wavenumber of a pattern of the liquid film observed in the experiment has the same parameter dependence as that of the fastest growth wavenumber.
\end{abstract}

Keywords: Marangoni dropwise condensation, Binary mixture, Stability analysis, Thin liquid film

\footnotetext{
*Tel. / Fax: +81-45-339-3897.

Email address: kentaro@ynu.ac.jp (Kentaro Kanatani)
} 


\section{Introduction}

When a binary vapor mixture of miscible liquids of some kind is condensed on a cooled substrate, the condensate liquid film often takes an inhomogeneous state such as a dropwise form. This phenomenon was first presented by Mirkovich and Missen [1]. Up to the present, a number of experiments on binary vapor condensation have been carried out and demonstrated nonfilmwise condensation (see Table 1 of Ref. [2] or Ref. [3]).

Ford and Missen [4] expressed the instability criterion of the condensate liquid film as

$$
\frac{\partial \sigma}{\partial h}>0
$$

where $\sigma$ and $h$ represent the surface tension and film thickness. Equation (1) corresponds to the case that the surface tension is stronger on a thicker part of the condensate than on a thinner one. In such a case, a stronger surface tension on a thicker portion pulls the liquid away from a thinner one and the deviation from the flat-film state is amplified. Since the surface tension is a function of temperature and concentration,

$$
\frac{\partial \sigma}{\partial h}=\left\{\frac{\partial \sigma}{\partial T_{I}}+\frac{\partial \sigma}{\partial c}\left(\frac{\partial c}{\partial T_{I}}\right)_{\text {sat }}\right\} \frac{\partial T_{I}}{\partial h},
$$

where $T_{I}$ and $c$ are the interface temperature and mass fraction of the component having a lower boiling point. The local thermodynamic equilibrium state is assumed at the liquid-vapor interface in evaluating $\left(\partial c / \partial T_{I}\right)_{\text {sat }}$ in Eq. (2), which is negative by the definition, unless the surface tension has a minimum or maximum point at an intermediate concentration. Since the substrate is cooled, the condition $\partial T_{I} / \partial h>0$ always holds. Furthermore, in most cases the dependence of surface tension on temperature is negligible compared to that on concentration. Therefore, the necessary condition for the condensate liquid film to be destabilized by the solutal Marangoni effect is

$$
\frac{\partial \sigma}{\partial c}<0
$$

which indicates that the surface tension of the high-boiling-point component is larger than that of the low-boiling-point component.

Stability of a flat-film state of the condensing liquid film in such a system was investigated by Hijikata et al. [5] However, they disregarded two 
interfacial boundary conditions necessary for condensation of binary mixtures: mass balance of the total mixture and that of a one component of the mixture. The first boundary condition entails mass gain of the liquid during the condensation process. It follows that Ref. [5] did not consider this effect. However, the experiments $[4,6]$ confirmed that there always exists a very thin layer of the liquid $(1 \mu \mathrm{m}$ order) between the condensate droplets, preventing the liquid film from rupturing. This observation is different from the theoretical consequence that an unstable liquid film subjected to only the destabilizing (thermal) Marangoni effect and the stabilizing gravity effect inevitably tends to rupture [7]. Moreover, since the length scale at which intermolecular forces between the liquid film and the solid surface influence the dynamics of the liquid-gas interface is about 10 to $100 \mathrm{~nm}$, it is found that the aforementioned very thin liquid layer between the droplets is not induced by any intermolecular forces, or is essentially irrelevant to the wettability of the solid surface. The above consideration implies that the effect of mass gain by condensation balances with that of solutocapillarity in such a length scale.

The second boundary condition which Hijikata et al. [5] ignored yields a relationship between the condensation mass flux and the concentration field. Since the liquid and vapor concentrations at the interface are determined by the interface temperature via the phase equilibrium condition, the mass flux becomes a function of the interface temperature. In Ref. [5] a similar relationship between the mass flux and the interface temperature is also formulated, assuming that the mass transfer coefficient is independent of temperature. However, if we allow for the second boundary condition, it can be shown that the mass transfer coefficient depends on temperature. Therefore, when the interface is irregular the value of the mass transfer coefficient locally varies owing to the variation of the interface temperature.

In this work, we first model the dynamics of a condensing liquid film of a binary vapor mixture. Applying the long-wave approximation [8] to the condensate liquid film, a nonlinear partial differential equation describing the spatio-temporal evolution of the film thickness is derived. The advantages of the use of such a low-dimensional approximate model are twofold: (i) to obtain analytical expressions for the dispersion relation of disturbances and for the stability criterion; and (ii) to reduce the computational amount in seeking time-dependent solutions at the strongly nonlinear regime. In the present paper, we focus on the linear stability of the flat-film state of the condensate, pertaining to the former advantage, and nonlinear calculations of the model, 
pertaining to the latter, shall be done subsequently. We incorporate the two boundary conditions mentioned above in deriving the model. The first boundary condition makes the basic state with a flat liquid-vapor interface time-dependent. Most studies on the stability of evaporating or condensing liquid layers (e.g. Ref. [9]) have treated the basic state as a steady state, assuming that the variation of the basic state is sufficiently slow compared to the growth rate of disturbances (the quasi-steady hypothesis). However, in the framework of the long-wave model the evolution equation of the amplitude of disturbances is easily integrable even when the average thickness of the liquid layer is varying by evaporation or condensation [10]. Reference [10] distinguished an algebraic behavior of the amplitude, arising from the mass loss or gain, from an exponential one and neglected the former. Thus the linear stability property provided by the long-wave model is more global in time. We also examine the influence of the dependence of the mass transfer coefficient on the interface temperature resulting from the second boundary condition.

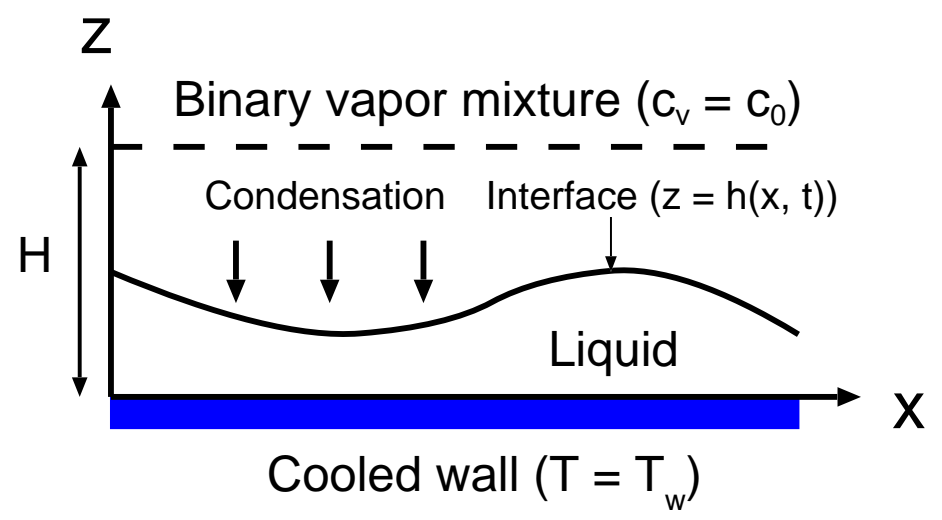

Figure 1: Sketch of a condensate liquid film of a binary vapor mixture on a cooled horizontal surface.

\section{Formulation}

\subsection{Governing equations}

We consider a two-dimensional condensate liquid film of a binary vapor mixture on a horizontal wall uniformly cooled at a temperature $T_{w}$, as depicted in Fig. 1. The $x$-axis coincides with the liquid-solid boundary and 
the $z$-axis points vertically upward to the wall. The mass fraction of the surrounding vapor is fixed to $c_{0}$ at $z=H$; here we assume that $H$ is much larger than the thickness of the condensate and never affected by the variation of the latter. The governing equations of the liquid phase are the continuity, Navier-Stokes, energy and mass transport equations:

$$
\begin{aligned}
& \nabla \cdot \boldsymbol{v}=0 \\
& \rho\left(\boldsymbol{v}_{t}+\boldsymbol{v} \cdot \nabla \boldsymbol{v}\right)=-\nabla p+\eta \nabla^{2} \boldsymbol{v}-\rho g \boldsymbol{e}_{z} \\
& T_{t}+\boldsymbol{v} \cdot \nabla T=\kappa \nabla^{2} T \\
& c_{t}+\boldsymbol{v} \cdot \nabla c=D \nabla^{2} c
\end{aligned}
$$

where $\boldsymbol{v}, p, T$ and $c$ are the velocity, pressure, temperature and mass concentration fields, respectively, inside the condensate. The differential operator is $\nabla \equiv\left(\partial_{x}, \partial_{z}\right)$. The symbols $\rho, \eta, \kappa$ and $D$ denote the density, dynamic viscosity, thermal diffusivity and mass diffusivity of the liquid, respectively, all assumed to be constant, $g$ the gravitational acceleration, and $\boldsymbol{e}_{z}$ the unit vector in the $z$ direction. Here we have neglected buoyancy, thermo-diffusion and diffusion-thermo effects.

We impose the no-slip, no-penetration, constant temperature and reflecting concentration boundary conditions at the wall,

$$
\boldsymbol{v}=\mathbf{0}, \quad T=T_{w}, \quad c_{z}=0 \quad \text { at } \quad z=0 .
$$

At the liquid-vapor interface $(z=h(x, t))$, the mass balance condition is expressed as

$$
J=\rho\left(\boldsymbol{v}_{I} \cdot \boldsymbol{n}-\boldsymbol{v} \cdot \boldsymbol{n}\right),
$$

where $J$ is the condensation mass flux, $\boldsymbol{n}$ the upward-directed unit vector normal to the interface,

$$
\boldsymbol{n}=\frac{\left(-h_{x}, 1\right)}{\sqrt{1+\left(h_{x}\right)^{2}}}
$$

and $\boldsymbol{v}_{I}$ the interface velocity, which satisfies the kinematic condition,

$$
\boldsymbol{v}_{I} \cdot \boldsymbol{n}=\frac{h_{t}}{\sqrt{1+\left(h_{x}\right)^{2}}} .
$$

Equation (9) was not taken into account in Ref. [5]. We assume the continuity of the interfacial stress and energy:

$$
\begin{gathered}
\left(p-p_{0}\right) \boldsymbol{n}-2 \eta \boldsymbol{E} \cdot \boldsymbol{n}+2 \sigma K \boldsymbol{n}+(\boldsymbol{t} \cdot \nabla \sigma) \boldsymbol{t}=\mathbf{0}, \\
J L=\lambda \nabla T \cdot \boldsymbol{n}
\end{gathered}
$$


where $p_{0}$ is the ambient vapor pressure, $\boldsymbol{E}$ the rate-of-strain tensor in the liquid phase, $\sigma$ the surface tension, $K$ the mean curvature of the interface,

$$
2 K=\frac{h_{x x}}{\left\{1+\left(h_{x}\right)^{2}\right\}^{3 / 2}}
$$

$\boldsymbol{t}$ the unit tangent vector to the interface,

$$
\boldsymbol{t}=\frac{\left(1, h_{x}\right)}{\sqrt{1+\left(h_{x}\right)^{2}}},
$$

$L$ the latent heat and $\lambda$ the thermal conductivity of the liquid. Note that the effects of vapor recoil, stress induced by the vapor motion, heat flux in the gas phase and viscous dissipation are neglected in Eqs. (12) and (13). As in Ref. [5], we postulate local thermodynamic equilibrium at the interface, which relates the local concentrations to the interface temperature $T_{I}$ :

$$
\begin{aligned}
c & =f\left(T_{I}\right), \\
c_{v} & =f_{v}\left(T_{I}\right) .
\end{aligned}
$$

Here and hereafter the subscript $v$ represents the quantity referring to the vapor phase. In this study, Eqs. (16) and (17) are linearized around the wall temperature,

$$
\begin{aligned}
c & =f\left(T_{w}\right)+f^{\prime}\left(T_{w}\right)\left(T_{I}-T_{w}\right), \\
c_{v} & =f_{v}\left(T_{w}\right)+f_{v}^{\prime}\left(T_{w}\right)\left(T_{I}-T_{w}\right) .
\end{aligned}
$$

Regarding the surface tension, we use the linear approximation for the dependence on the temperature and the liquid concentration at the interface:

$$
\begin{aligned}
\sigma & =\sigma_{0}+\left.\frac{\partial \sigma}{\partial T}\right|_{T=T_{w}}\left(T_{I}-T_{w}\right)+\left.\frac{\partial \sigma}{\partial c}\right|_{T=T_{w}}\left(c-f\left(T_{w}\right)\right) \\
& =\sigma_{0}+\left(\frac{\partial \sigma}{\partial T}+\frac{\partial \sigma}{\partial c} f^{\prime}\right)_{T=T_{w}}\left(T_{I}-T_{w}\right)
\end{aligned}
$$

where Eq. (18) has been substituted. Finally, in order to evaluate the condensation mass flux we utilize the mass flux balance of one component of the binary mixture at the surface,

$$
c J=\rho_{v} D_{v} \nabla c_{v} \cdot \boldsymbol{n}+c_{v} J,
$$


where we have neglected the concentration gradient term of the liquid phase, since the mass diffusivity of liquid is usually much smaller than that of gas. Equation (21) can be rewritten into the expression for $J$ and linearized with respect to the temperature as

$$
\begin{aligned}
J & =-\frac{\rho_{v} D_{v} \nabla c_{v} \cdot \boldsymbol{n}}{c_{v}-c} \\
& =J_{0}-\left.\frac{d}{d T} \frac{\rho_{v} D_{v} \nabla c_{v} \cdot \boldsymbol{n}}{c_{v}-c}\right|_{T=T_{w}}\left(T_{I}-T_{w}\right) .
\end{aligned}
$$

This equation includes the gradient of the vapor concentration, which cannot be known without solving the bulk vapor phase equations. However, in order to simplify the problem it is modeled as

$$
\left.\nabla c_{v} \cdot \boldsymbol{n}\right|_{z=h}=\frac{c_{0}-f_{v}\left(T_{I}\right)}{\delta}
$$

where $\delta$ is a phenomenological parameter of dimension of length determined from the ambient-vapor condition. Equation (23) is an analogue of wellknown Newton's law of cooling for heat transfer.

Here we note that in general the concentration distribution in the gas phase can vary with time. In Fig.10 of Ref. [11] the vapor concentration is set uniform at the bulk value at the initial time, which corresponds to the time when subcooling is commenced. As time progresses, the parameter $\delta$, associated with the vertical differential of the vapor concentration, increases from the initial time. This initial transient behavior complicates the modeling. For simplicity, in this paper we restrict our attention to cases where $\delta$ takes a saturated value after some period from the commencement of subcooling. In the experiment [5], the cooling surface was periodically wiped to remove the condensate. When the variation of the vapor concentration field becomes slow enough for $\delta$ to be regarded as independent of time, our model can be applied.

It is stressed that keeping $\delta$ constant and keeping the location at which the vapor concentration is fixed constant is not the same when the wall temperature or the mass fraction of the ambient vapor is changed. In order to see this, we first solve the one-dimensional stationary convection-diffusion equation of the gas phase in the vertical direction,

$$
-v_{v} c_{v z}=D_{v} c_{v z z}
$$


with boundary conditions $c_{v}=f_{v}\left(T_{w}\right)$ at $z=0$ and $c_{v}=c_{0}$ at $z=H$. The solution is

$$
c_{v}=c_{0}+\left\{f_{v}\left(T_{w}\right)-c_{0}\right\} \frac{e^{v_{v}(H-z) / D_{v}}-1}{e^{v_{v} H / D_{v}}-1} .
$$

It is noted that this distribution is valid only if the variables are horizontally uniform. When the liquid-vapor interface is deformed, the vapor concentration at the interface varies from the phase equilibrium relation (17) according to the fluctuation of the interface temperature and then the effect of the concentration diffusion in the horizontal direction cannot be neglected. This is the reason why we adopt the phenomenological description (23) for the vaporconcentration derivative term in Eq. (22). Substitution of the distribution (25) into Eq. (23) yields

$$
\delta=\frac{D_{v}}{v_{v}}\left(1-e^{-v_{v} H / D_{v}}\right)
$$

Since the vapor velocity $v_{v}$ is related to the mass flux through $J=\rho_{v} v_{v}$, the expression for $v_{v}$ is obtained from Eq. (21) as

$$
v_{v}=\frac{D_{v}}{H} \log \left(\frac{f_{v}-f}{c_{0}-f}\right)_{T=T_{w}} .
$$

From Eqs. (26) and (27)

$$
\delta=\left.\frac{H}{\log \left(\frac{f_{v}-f}{c_{0}-f}\right)}\left(\frac{f_{v}-c_{0}}{f_{v}-f}\right)\right|_{T=T_{w}} .
$$

Thus the relationship between $\delta$ and $H$ is dependent on the wall temperature and the ambient-vapor concentration. In the analysis below, we use $H$ instead of $\delta$ as a control parameter.

Above are all of the boundary conditions which we use. However, it is worthwhile noting here a difference from the earlier theoretical work [5]. The authors of Ref. [5] assumed that the condensation mass flux is proportional to the difference between the vapor mass concentration at the interface and the ambient one:

$$
J=\beta\left\{f_{v}\left(T_{I}\right)-c_{0}\right\},
$$


where $\beta$ is the mass transfer coefficient. This equation was further linearized with respect to the temperature under the phase equilibrium relation as follows:

$$
J=\beta f_{v}^{\prime}\left(T_{I}-T_{0}\right),
$$

where $T_{0}$ is the ambient temperature. Nevertheless, in their formulation (30) the temperature dependence of the mass transfer coefficient $\beta$ itself was not considered. In fact, $\beta$ does depend on the temperature. If we substitute Eq. (23) into Eq. (22), it is found that the mass transfer coefficient $\beta$ corresponds to $\rho_{v} D_{v} / \delta\left(f_{v}-f\right)_{T=T_{I}}$ by comparison between Eqs. (22) and (29). Even if we regard the material properties $\rho_{v}$ and $D_{v}$ as constant, $\left(f_{v}-f\right)_{T=T_{I}}$ varies with the interface temperature. In our model, we take into account the effect of the temperature variation of the mass transfer coefficient through the local thermodynamic equilibrium assumption.

\subsection{Nondimensionalization}

We scale lengths, time, velocities and pressures by $d, d^{2} \rho / \eta, \eta / d \rho$ and $\eta^{2} / d^{2} \rho$, respectively, where $d$ is the characteristic film thickness. Note that the film thickness increases during condensation. However, $d$ is defined as a proper constant and never varies throughout the temporal evolution of the liquid film. A mass flux scale is chosen from Eqs. (22) and (23) as

$$
J_{0}=\frac{\rho_{v} D_{v}}{\delta}\left(\frac{f_{v}-c_{0}}{f_{v}-f}\right)_{T=T_{w}}=\frac{\rho_{v} D_{v}}{H} \log \left(\frac{f_{v}-f}{c_{0}-f}\right)_{T=T_{w}},
$$

where Eq. (28) has been substituted. Thus it is natural from Eq. (13) to nondimensionalize temperature by

$$
\Delta T=\frac{d L J_{0}}{\lambda}=\frac{d L \rho_{v} D_{v}}{\lambda H} \log \left(\frac{f_{v}-f}{c_{0}-f}\right)_{T=T_{w}} .
$$

We note that $\Delta T$ can be interpreted as the temperature difference across a flat motionless condensate of thickness $d$. Therefore, if one can measure from an experiment the interface and wall temperatures and the film thickness when the condensate is flat, the value of $H$ can be assessed from Eq. (32). The 
above nondimensionalization yields the following dimensionless parameters:

$$
\begin{aligned}
G & =\frac{g d^{3} \rho^{2}}{\eta^{2}} \\
S & =\frac{\sigma_{0} d \rho}{\eta^{2}} \\
\Gamma & =\frac{d J_{0}}{\eta}=\frac{\lambda \Delta T}{\eta L}=\frac{d \rho_{v} D_{v}}{\eta H} \log \left(\frac{f_{v}-f}{c_{0}-f}\right)_{T=T_{w}}, \\
\Sigma & =\frac{\Delta T}{J_{0}}\left(\frac{d J}{d T}\right)_{T=T_{w}}=\left(-\frac{f_{v}^{\prime}}{f_{v}-c_{0}}+\frac{f_{v}^{\prime}-f^{\prime}}{f_{v}-f}\right)_{T=T_{w}} \Delta T \\
& =\left.\frac{d L \rho_{v} D_{v}}{\lambda H}\left(-\frac{f_{v}^{\prime}}{f_{v}-c_{0}}+\frac{f_{v}^{\prime}-f^{\prime}}{f_{v}-f}\right) \log \left(\frac{f_{v}-f}{c_{0}-f}\right)\right|_{T=T_{w}}, \\
M & =\frac{d \rho \Delta T}{\eta^{2}}\left(\frac{\partial \sigma}{\partial T}+\frac{\partial \sigma}{\partial c} f^{\prime}\right) T_{T=T_{w}} \\
& =\left.\frac{d^{2} \rho L \rho_{v} D_{v}}{\eta^{2} \lambda H}\left(\frac{\partial \sigma}{\partial T}+\frac{\partial \sigma}{\partial c} f^{\prime}\right) \log \left(\frac{f_{v}-f}{c_{0}-f}\right)\right|_{T=T_{w}} .
\end{aligned}
$$

Here $G, S, \Gamma, \Sigma$ and $M$ are the Galileo, inverse capillary, condensation, modified Sherwood and modified Marangoni numbers, respectively, and represent effects of gravity, surface tension, condensation mass flux, its temperature variation and thermo- or soluto-capillarity, respectively. The definition of the Sherwood number consists of two terms in the first pair of parentheses: if we take only the first one, it is identical with the definition of the Sherwood number in Ref. [5]; the second term reflects the effect of the temperature variation of the mass transfer coefficient.

\subsection{Long-wave model}

Assuming that the characteristic wavelength in the horizontal direction is much larger than the film thickness, we apply the long-wave approximation to the condensate. The procedure to obtain a single partial differential equation for the film thickness is rather classical, so that we omit it in this paper. For a detailed derivation of the model the reader should refer to, for example, Ref. [8]. However, a following point should be noted. Under the assumption of local thermodynamic equilibrium, the surface concentrations are uniquely prescribed by the temperature there, as in Eqs. (16) and (17). Therefore, solving the energy equation (6) is sufficient to describe the solutocapillary effect. Accordingly, the derived model does not include the equation for 
concentration (7). This is a significant difference from the other long-wave model of a binary liquid film [12] and the stability analysis of an evaporating binary liquid layer [9].

The final result becomes

$$
h_{t}=\left\{\frac{h^{3}}{3}\left(G h_{x}-S h_{x x x}\right)-\frac{M h^{2} h_{x}}{2(1+\Sigma h)^{2}}\right\}_{x}+\frac{\Gamma}{1+\Sigma h} .
$$

This equation itself is no novelty, since the similar forms of equation have already investigated extensively [8].

\section{Linear stability analysis}

Since the liquid film is condensing, its total mass increases with time. First we consider the basic state $\bar{h}(t)$ where the flat interface moves upward. By dropping the derivative of $x$ in the model equation (34) we obtain

$$
\bar{h}_{t}=\frac{\Gamma}{1+\Sigma \bar{h}} \text {. }
$$

Integration with an initial condition $\bar{h}(0)=\bar{h}_{0}$ gives

$$
\bar{h}=\frac{-1+\sqrt{\left(1+\Sigma \bar{h}_{0}\right)^{2}+2 \Gamma \Sigma t}}{\Sigma} .
$$

In order to seek the stability property of $\bar{h}(t)$, we employ the normal mode ansatz

$$
h(x, t)=\bar{h}(t)+A(k, t) \exp (i k x) \quad(|A| \ll \bar{h})
$$

for a disturbance of the wavenumber $k$. Substitution into Eq. (34) and linearization with respect to the amplitude of the disturbance $A$ provides the ordinary differential equation for $A$,

$$
\frac{A_{t}}{A}=-k^{2}\left\{\frac{\bar{h}^{3}}{3}\left(G+S k^{2}\right)-\frac{M \bar{h}^{2}}{2(1+\Sigma \bar{h})^{2}}\right\}-\frac{\Gamma \Sigma}{(1+\Sigma \bar{h})^{2}} .
$$

Integrating this with Eq. (35), we obtain

$$
\begin{aligned}
& A=A(t=0) \\
&\left(\frac{1+\Sigma \bar{h}_{0}}{1+\Sigma \bar{h}}\right)^{1-M k^{2} / 2 \Gamma \Sigma^{3}} \exp \left[\frac { k ^ { 2 } ( \overline { h } - \overline { h } _ { 0 } ) } { \Gamma } \left\{\frac{M}{4 \Sigma}\left(\bar{h}+\bar{h}_{0}-\frac{2}{\Sigma}\right)\right.\right. \\
&\left.\left.-\frac{G+S k^{2}}{3}\left(\frac{\bar{h}_{0}^{3}}{4} \sum_{i=0}^{3}\left(\frac{\bar{h}}{\bar{h}_{0}}\right)^{i}+\frac{\Sigma \bar{h}_{0}^{4}}{5} \sum_{i=0}^{4}\left(\frac{\bar{h}}{\bar{h}_{0}}\right)^{i}\right)\right\}\right] .
\end{aligned}
$$


If we take the limit of $\bar{h}_{0} \rightarrow 0$,

$$
\begin{gathered}
A=A(t=0) \exp \left[-\log (1+\Sigma \bar{h})+\frac{M k^{2}}{4 \Gamma \Sigma^{3}}\{2 \log (1+\Sigma \bar{h})+\Sigma \bar{h}(\Sigma \bar{h}-2)\}\right. \\
\left.-\frac{G k^{2}+S k^{4}}{3 \Gamma}\left(\frac{\bar{h}^{4}}{4}+\frac{\Sigma \bar{h}^{5}}{5}\right)\right] .
\end{gathered}
$$

The Marangoni instability emerges at long wavelengths $(k \ll 1)$ while disturbances of short wavelengths $(k \gg 1)$ decay due to the effect of surface tension. Note that the Marangoni term in the exponent in Eq. (40) is positive for any film thickness. Since the condensation number $\Gamma$ is often much less than the other parameters, we may neglect the algebraic coefficient $(1+\Sigma \bar{h})^{-1}$ in Eq. (40), which arises from the mass-gain term in Eq. (38). The cutoff wavenumber $k_{c}$ and the fastest growth wavenumber $k_{\max }$ are given by equating the exponent in Eq. (40) to zero and setting $A_{k}=0$, respectively:

$$
\begin{gathered}
k_{c}=\sqrt{\frac{15 M \Sigma}{S}\left\{\frac{2 \log (1+\Sigma \bar{h})+\sum \bar{h}(\Sigma \bar{h}-2)}{5(\Sigma \bar{h})^{4}+4(\Sigma \bar{h})^{5}}\right\}-\frac{G}{S}}, \\
k_{\max }=\frac{k_{c}}{\sqrt{2}} .
\end{gathered}
$$

Both cutoff and fastest growth wavenumbers are monotonically decreasing functions of the film thickness and diverge at zero film thickness. This fact apparently contradicts the long-wave assumption, on the basis of which our model was constructed. However, the relative value of the cutoff wavenumber with respect to the film thickness is expressed by their product:

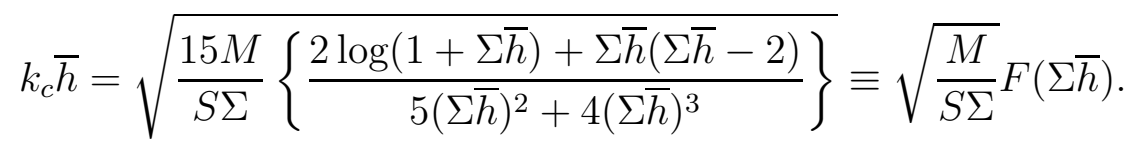

Here we have neglected the gravity effect $(G=0)$ for simplicity. Figure 2 shows the value of $F(x)$ in Eq. (43). The function $F(x)$ converges to zero in the limit of $x \rightarrow 0$ and takes the maximum value around 0.811 at $x \simeq 1.35$. Therefore, although the absolute value of the cutoff wavenumber diverges as the film thickness tends to zero, the ratio of the film thickness to an unstable wavelength of the disturbance vanishes. 


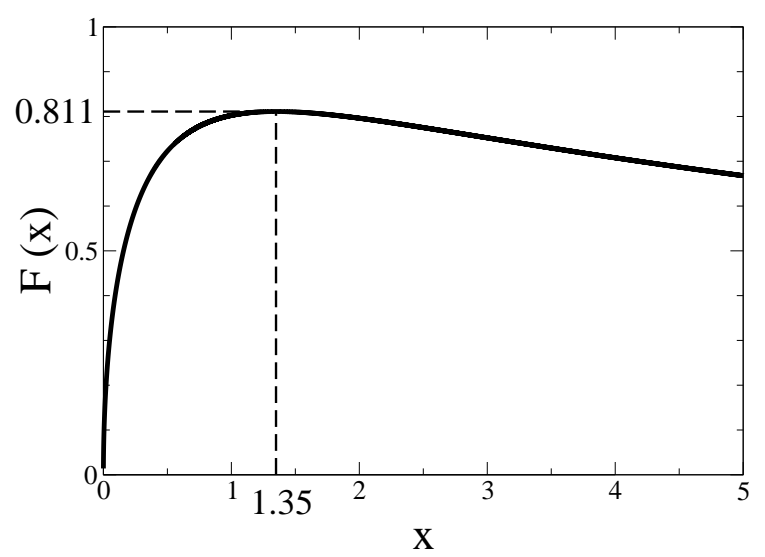

Figure 2: The value of $F(x)$ in Eq. (43).

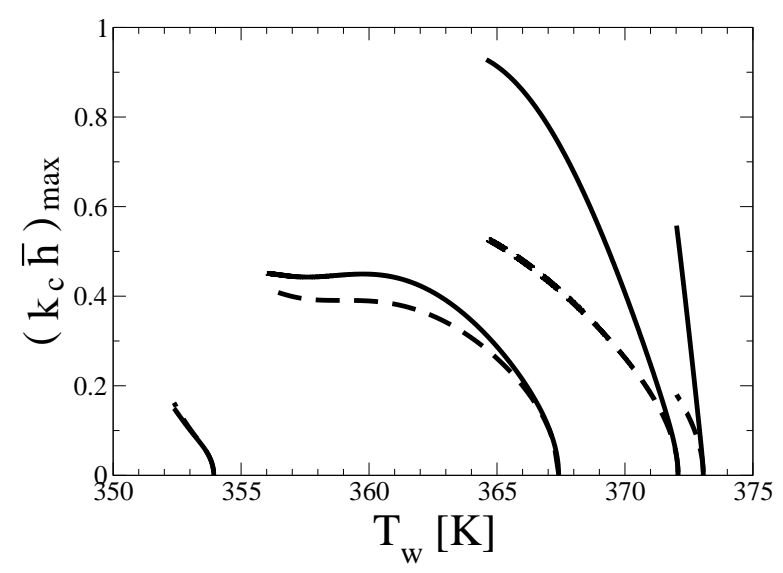

Figure 3: The value of $\left(k_{c} \bar{h}\right)_{\max } \simeq 0.811 \sqrt{M /(S \Sigma)}$ versus the wall temperature $T_{w}$ for water-ethanol mixture at atmospheric pressure. The solid curves represent the values obtained from our definition of the Sherwood number (33) for correspond to the cases for $c_{0}=0.01,0.1,0.4$ and 0.8 from right to left. The dashed curves indicate the corresponding cases where the temperature dependence of the mass transfer coefficient is ignored. Each pair of the curves is plotted in the temperature ranges satisfying $f\left(T_{w}\right)<c_{0}<f_{v}\left(T_{w}\right)$ (see Fig. 5). 


\subsection{Application to water-ethanol mixture}

In this subsection, we estimate the value of $\left(k_{c} \bar{h}\right)_{\max } \simeq 0.811 \sqrt{M /(S \Sigma)}$ for water-ethanol binary vapor mixture at atmospheric pressure, which has been frequently used in experiments $[3,6,11]$. The aim of this estimation is to verify the long-wave nature of the instability of the condensate liquid film and validate the use of our model. Figure 3 displays the value of $\left(k_{c} \bar{h}\right)_{\max }$ as a function of the wall temperature $T_{w}$ for different mass fractions of the ambient-vapor mixture. Note here that both $M$ and $S \Sigma$ are proportional to $d^{2} / H$ from their definitions (33) and hence $\left(k_{c} \bar{h}\right)_{\max }$ is determined only from the wall temperature and the ambient-vapor concentration. The values of the physical and phase-equilibrium properties are obtained in the way described in the Appendix. Although the Marangoni and Sherwood numbers diverge at the liquid line temperature indicated in Fig. $5\left(f\left(T_{w}\right)=c_{0}\right.$; the left edges of the curves in Fig. 3), $\left(k_{c} \bar{h}\right)_{\max }$ remains finite because the logarithmic function in the definitions of the Marangoni and Sherwood numbers (33), responsible for the divergence, cancels. All curves plotted in Fig. 3 satisfy the condition $\left(k_{c} \bar{h}\right)_{\max }<1$. Since the actual value of the ratio of the film thickness to the shortest unstable wavelength of the disturbance is $\left(k_{c} \bar{h}\right)_{\max } /(2 \pi)$, we confirmed that the ratio is $\mathcal{O}\left(10^{-1}\right)$ at most for a water-ethanol system.

We also address the value of the film thickness where $k_{c} \bar{h}$ takes the largest value, which is represented by $\bar{h}_{\max } \simeq 1.35 / \Sigma$. Since the Sherwood number is proportional to $d / H$ (see the definition (33)), dividing $\bar{h}_{\text {max }}$ by $H / d$ eliminates the dependence on both $d$ and $H$. This procedure corresponds to the change of the scaling of the dimensional film thickness $\bar{h}_{\text {max }}^{d}$ from $d$ to $H$. Figure 4 depicts the value of $\bar{h}_{\max }^{d} / H=\bar{h}_{\max } d / H$ for a water-ethanol system. Note that whereas $\bar{h}_{\max }$ vanishes in the limit of the liquid line temperature $f\left(T_{w}\right) \rightarrow c_{0}$, it takes a finite value at the vapor line temperature $\left(f_{v}\left(T_{w}\right)=c_{0}\right.$; the right edges of the curves) since the Sherwood number has the limit value

$$
\left.\Sigma\right|_{f_{v}\left(T_{w}\right) \rightarrow c_{0}}=-\frac{d L \rho_{v} D_{v}}{\lambda H} \frac{f_{v}^{\prime}}{f_{v}-f}
$$

Both in Figs. 3 and 4, we have also plotted the data calculated with the definition of the Sherwood number of Ref. [5], where the temperature dependence of the mass transfer coefficient is ignored, as the dashed curves for reference. In both figures a significant difference can be observed for low-ethanol concentrations of the ambient vapor. This difference becomes small for large fractions of ethanol of the ambient vapor (for $c_{0}=0.8$ it is 


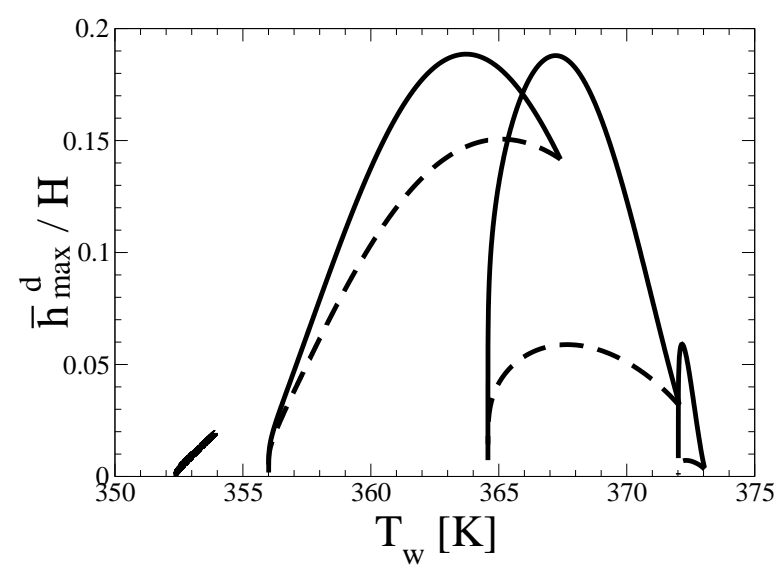

Figure 4: The film thickness where $k_{c} \bar{h}$ takes the largest value, scaled by $H, \bar{h}_{\max }^{d} / H=$ $\bar{h}_{\text {max }} d / H \simeq 1.35 d / \Sigma H$, versus the wall temperature $T_{w}$ for water-ethanol mixture at atmospheric pressure. The solid curves represent the values obtained from our definition of the Sherwood number (33) for $c_{0}=0.01,0.1,0.4$ and 0.8 from right to left. The dashed curves indicate the corresponding cases where the temperature dependence of the mass transfer coefficient is ignored. Each pair of the curves is plotted in the temperature ranges satisfying $f\left(T_{w}\right)<c_{0}<f_{v}\left(T_{w}\right)$ (see Fig. 5).

indiscernible). Each pair of the curves has the same value at the vapor line temperature $\left(f_{v}\left(T_{w}\right)=c_{0}\right)$, because the term representing the temperature dependence of the mass transfer coefficient disappears in the definition of the Sherwood number in this limit (see Eq. (44)). Where the wall temperature is near the boiling point of water or ethanol (for $c_{0}=0.01$ or 0.8 ), the values of $\left(k_{c} \bar{h}\right)_{\max }$ and $\bar{h}_{\text {max }}$ are smaller than those for the other concentrations. In this region, the vapor and liquid lines in Fig. 5 approach each other $\left(f_{v}\left(T_{w}\right) \sim f\left(T_{w}\right)\right)$. Then the denominators $f_{v}\left(T_{w}\right)-c_{0}$ and $f_{v}\left(T_{w}\right)-f\left(T_{w}\right)$ in the definition of the Sherwood number (33) is reduced, which decreases the values of $\left(k_{c} \bar{h}\right)_{\max } \propto \Sigma^{-1 / 2}$ and $\bar{h}_{\max } \propto \Sigma^{-1}$.

Finally a comparison with the experimental results is discussed. The wavenumber of a pattern on the condensate liquid film observed in experiments is considered to have the same parameter dependence as that of the fastest growth wavenumber $k_{\max }$ in Eq. (42). In Fig. 8 of Ref. [5], the authors plotted their experimental data of the wavenumber of the pattern on the condensate divided by the Marangoni number (in their definition) over the Prandtl number against the Marangoni number over the Prandtl number, 
since they conjectured that the wavenumber of the pattern would be proportional to the Marangoni number divided by the Prandtl number. However, the data points deviate from their conjecture toward lower wavenumbers. In our model, the fastest growth wavenumber is proportional to the square root of the Marangoni number if the gravity effect is neglected (see Eq. (41)). The data plots appear to be better fitted with the line of the square root of the Marangoni number rather than that of the Marangoni number. Although the definition of the Marangoni number (the temperature difference) is different between theirs and ours, the expression of the fastest growth wavenumber obtained from our model would explain the Marangoni number dependence of the experimental results. The deviation from the square root of the Marangoni number might be attributed to the effects of variations of the other parameters ( $\Sigma$ or $S$ ). The precise value of the wavenumber of the pattern cannot be predicted only by the linear theory, since the selected wavenumber is determined according to the film thickness where nonlinearity emerges.

\section{Conclusions}

We have derived a low-dimensional model describing the dynamics of a condensing liquid film in a binary vapor mixture system by applying the long-wave approximation. In the modeling, we have adopted an interfacial boundary condition taking account of an effect of mass gain, which was not considered in the earlier theoretical study [5]. Furthermore, in Ref. [5] the mass transfer coefficient is assumed to be independent of temperature. However, we have shown that it indeed depends on temperature through the condition of mass flux balance of a one component of the mixture and the phase equilibrium relation at the liquid-vapor interface. We have incorporated the temperature derivative of the mass transfer coefficient into the definition of the Sherwood number, which represents an effect of the temperature variation of condensation mass flux in this study. In the expression of the condensation mass flux, the vapor concentration gradient term has been assumed to be proportional to the concentration difference between the interface and the ambient vapor. For simplicity we have treated its proportionality coefficient as time-independent. However it varies with the wall temperature and the ambient-vapor concentration once the location of the fixed vapor concentration is settled.

We have conducted a linear stability analysis of a flat-film state of the 
condensate based on the derived model. As a consequence of the inclusion of the effect of mass gain, the flat liquid-vapor interface is moving upward by condensation in the basic state. We have obtained the evolution equation of the amplitude of a disturbance, from which the cutoff and fastest growth wavenumbers are deduced. Both wavenumbers are functions of the film thickness and diverge in the limit of zero film thickness. However, we have shown that their relative values with respect to the film thickness vanishes in the same limit.

We have evaluated the maximum value of the relative cutoff wavenumber for water-ethanol binary vapor mixture at atmospheric pressure. We have numerically demonstrated the long-wave nature of the instability of the condensate liquid film in this system. We have also assessed the film thickness where the ratio of the film thickness to the wavelength of an unstable disturbance takes the maximum value in the same system. Since both the maximum relative cutoff wavenumber and its film thickness are dependent on the Sherwood number, their values are affected by the temperature dependence of the mass transfer coefficient. We have found that this effect cannot be neglected for a water-ethanol system of small ethanol fractions of the ambient vapor. The values of the maximum relative cutoff wavenumber and its film thickness are reduced as the wall temperature approaches the boiling points of the pure components of the mixture from the decrease of the Sherwood number. Finally, we have shown that the wavenumber of a pattern on the condensate liquid film appeared in the experiment [5] has the same parameter dependence as that of the fastest growth wavenumber predicted by our model. In order to obtain the concrete value of the wavenumber of the pattern, nonlinear computations should be performed.

\section{Acknowledgements}

The author gratefully acknowledges Hiroaki Matsumoto, who provided the doctor thesis of Zhihao Chen and an opportunity to do this research, Yoshio Utaka and the reviewer of this article for their invaluable and insightful comments on this work.

\section{Appendix. Physical properties}

The values of the physical and phase-equilibrium properties of waterethanol mixture at atmospheric pressure except the surface tension are cal- 


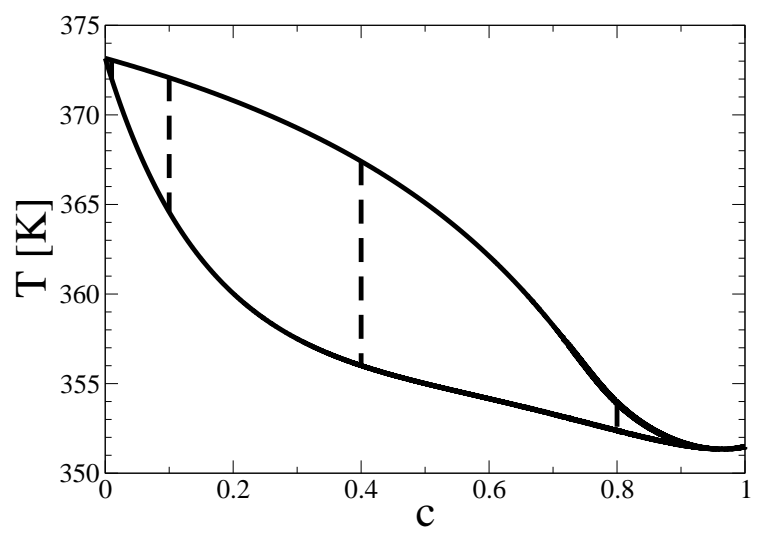

Figure 5: The liquid-vapor phase equilibrium diagram for water-ethanol mixture at atmospheric pressure, calculated from the formulae written in Ref. [13]. The lower and upper solid curves are the liquid and vapor lines $\left(f(T)=c\right.$ and $f_{v}(T)=c$ in Eqs. (16) and (17)). The dashed lines indicate the domains of the wall temperature for $c_{0}=0.01,0.1,0.4$ and 0.8, where the data in Figs. 3 and 4 are plotted.

culated from the formulae written in Ref. [13]. The surface tension is extrapolated from the experimental data between 20 and $50^{\circ} \mathrm{C}$ [14] to the temperature on the liquid line $(f(T)=c$ in Eq. (16)) in the liquid-vapor phase equilibrium diagram (Fig. 5) for each concentration at atmospheric pressure. We interpolate the extrapolated points of the surface tension with respect to the concentration and neglect its temperature dependence. All of the properties are evaluated at the wall temperature $T_{w}$.

Note that some experiments $[11,15]$ showed that the condensate took a droplet form below the liquid line, where the vapor mixture is supposed to condensate at the same concentration (local total condensation). Since we assume local thermodynamic equilibrium at the interface into our model, this region is out of the scope of applicability of our model. Although we do not know even whether or not the interfacial nonequilibrium is actually attained when the wall temperature is below the liquid line, it is reasonable to relax the equilibrium condition and impose a nonequilibrium one to model the condensate in the state of local total condensation. However, to our knowledge there is no existing theory for describing an interfacial nonequilibrium state of binary mixtures.

[1] V. V. Mirkovich, R. W. Missen, Non-filmwise condensation of binary vapors of miscible liquids, Canadian J. Chem. Eng. 39 (1961) 86-87. 
[2] Y. Li, J. Yan, J. Wang, G. Wang, A semi-empirical model for condensation heat transfer coefficient of mixed ethanol-water vapors, J. Heat Transfer 133 (2011) 061501-1-061501-11.

[3] X. Ma, Z. Lan, W. Xu, M. Wang, S. Wang, Effect of surface free energy difference on steam-ethanol mixture condensation heat transfer, Int. J. Heat Mass Transfer 55 (2012) 531-537.

[4] J. D. Ford, R. W. Missen, On the conditions for stability of falling films subject to surface tension disturbances; the condensation of binary vapor, Canadian J. Chem. Eng. 46 (1968) 309-312.

[5] K. Hijikata, Y. Fukasaku, O. Nakabeppu, Theoretical and experimental studies on the pseudo-dropwise condensation of a binary vapor mixture, J. Heat Transfer 118 (1996) 140-147.

[6] Y. Utaka, T. Nishikawa, Measurement of condensate film thickness for solutal marangoni condensation applying laser extinction method, J. Enhanced Heat Transfer 10 (2003) 119-130.

[7] A. Oron, P. Rosenau, Formation of patterns induced by thermocapillarity and gravity, J. Phys. II 2 (1992) 131-146.

[8] A. Oron, S. H. Davis, S. G. Bankoff, Long-scale evolution of thin liquid films, Rev. Mod. Phys. 69 (1997) 931-980.

[9] H. Machrafi, A. Rednikov, P. Colinet, P. C. Dauby, Bénard instabilities in a binary-liquid layer evaporating into an inert gas, J. Colloid Interface Sci. 349 (2010) 331-353.

[10] J. P. Burelbach, S. G. Bankoff, S. H. Davis, Nonlinear stability of evaporating/condensing liquid films, J. Fluid Mech. 195 (1988) 463-494.

[11] Y. Utaka, S. Wang, Characteristic curves and the promotion effect of ethanol addition on steam condensation heat transfer, Int. J. Heat Mass Transfer 47 (2004) 4507-4516.

[12] M. Bestehorn, I. D. Borcia, Thin film lubrication dynamics of a binary mixture: Example of an oscillatory instability, Phys. Fluids 22 (2010) 104102-1-104102-10. 
[13] T. Fujii, Theory of Laminar Film Condensation, Springer-Verlag, New York, 1991.

[14] G. Vázquez, E. Alvarez, J. M. Navaza, Surface tension of alcohol + water from 20 to $50{ }^{\circ} \mathrm{C}$, J. Chem. Eng. Data 40 (1995) 611-614.

[15] J. N. A. Morrison, J. Deans, Augmentation of steam condensation heat transfer by addition of ammonia, Int. J. Heat Mass Transfer 40 (1997) $765-772$. 